\title{
Brainwave Detection Model for Panic Attacks Based on Event-related Potential
}

\author{
Puwadol Sirikongtham ${ }^{1}$, Worapat Paireekreng ${ }^{2}$ and Suwannit Chareen Chit $^{3}$ \\ ${ }^{1,2}$ Dhurakij Pundit University, Thailand \\ ${ }^{3}$ Universiti Utara Malaysia, Malaysia
}

${ }^{1}$ puwadol.sirikongtham@dpu.ac.th; ${ }^{2}$ worapat.png@dpu.ac.th; ${ }^{3}$ chareen@uum.edu.my

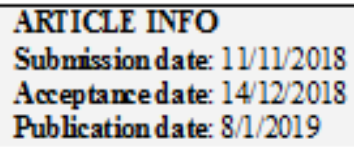

\begin{abstract}
Panic attacks could adversely affect a patient's daily life and can pose risks to others. The symptoms of panic attacks can be timely observed by detecting the brainwave. This research presents a model that can evaluate the level of panic attack symptoms using the brainwaves detection during (or before) the symptom occurs. It helps monitor the patient's brainwave based on Event-related potential (ERP). The model is derived from the simulation with horror pictures and frightening sound on the experimental group of 30 people. The survey related to symptoms which has been used regarding to the criteria of the Beck Anxiety Inventory (BAI). The results showed that there is a consistent change of Electroencephalography (EEG) in each change of brainwaves where its quantitative analysis found that the changes of Beta, Gamma, and Alpha directly affect the model of Brainwaves Panic Attacks Measurement (BPAM) which is associated with panic attacks. 1 out of 30 cases scored higher than the average of the BPAM at 220 The Model BPAM can detect the risk to be Panic Attack compared to the use of tests Beck Anxiety Inventory (BAI) were found to be consistent. The test value BAI Score 1963 was BPAM Score 401-1000. In addition, the results found that at P300 the brainwave pattern of EEG in meditation had decreased significantly whereas the brainwave related to attention had increased considerably for which human brain can potentially respond to stimulated external events.
\end{abstract}

Keywords: Beck Anxiety Inventory (BAI), brainwave patterns, event-related potential, panic attacks.

\section{Introduction}

Brainwaves have been a medical topic of interest for a long time. Studies have been conducted to determine the cause and prevent diseases related to the brain. Recent advancement in information and communication technology have paved ways for biomedical signal processing, making it possible to analyses signals from human bodies and study brainwaves. This has led to the development of brainwaves detection and analysis, and has gained tremendous research attention in the recent years [1].

Information and communication technology presents novel methods for detecting brainwaves. Doctors can now communicate with speech impaired patients using the electroencephalogram (EEG) by analyzing the characteristics of brain electric wave signals [2]. Some studies also suggest the use of brainwaves for patients suffering from muscle weakness disease, such as muscular dystrophy and inflammatory myopathy. The Necomimi [3] cat ears are the example to show emotion 
based on brainwaves detection which move the ears to represent the feelings of the wearer at that time. Puzzlebox Orbit helicopter can use the brainwaves to control the direction by moving up and down [4].

Human-machine communication using brainwaves (Brain Computer Interface: BCI) [5] can be applied to help people with disability (PWD). PWD can operate and control computer equipment or appliances through brainwaves detection. This technology also can be applied to facilitate people by analyzing electrical signals generated from movement of the muscles. The brainwaves signal is derived from brain neurons electromagnetic signals in the body. This signal is analyzed and transformed into digital form. Computers can understand the meaning of these signals and use it to interact with humans. There are various areas of BCI usage such as medical, industrial, or entertainment [6].

Humans normally respond to events through the autonomic nervous system which balances the homeostasis. Humans cannot control factors such as heartbeat, perspiration, or breathing. In contrast, the body system will run automatically when the external environment changes [7]. This results in increased heart-rate, heavy breathing, perspiration, and other responses to react to the situation which can be either good, or bad. The body will react to a stimulation. However, if there is too much system response towards stimuli, it automatically becomes panic attacks where signals are transmitted from the brain to organs to respond to the stimuli. Succession of panic attacks may result in a change or imbalance of neurotransmitters in the brain of patients. In addition, if panic attacks relapse often with the continuous fear, it will cause crucial impact in the patient's daily life [8].

Panic Attack symptoms may occur at any time due to stress. People living under a stressful life over a long period of time may contract diseases such as depression, anxiety, high blood pressure, cardiac abnormalities, diabetes, and much more. In some cases, panic attack due to high stress level while performing other activities such as driving, which can be harmful to other people. Stringent medical examination must be carried out each time a patient displays symptoms of panic attack. However, the cost for such examination high usually high and there is currently no way to diagnose the symptom except via external observation only. Therefore, it is necessary to detect and evaluate the symptoms of panic attacks via other means. A possible approach is to detect and handle changes in brainwaves during the symptoms occurred with an effective method to examine the patient. This can help people suffering from stress significantly [9].

This research proposes a model for detecting and determining brainwaves patterns associated with Panic Attacks. The research conducts the experiment on the simulation with picture and sound which users test the experience whether there is Panic Attacks or not. They are tested about fear or shocking that is more than usual. The users will be captured the brainwaves signal during the experiment to find the Panic Attacks brainwaves.

\section{Background}

\section{Brainwaves Detection}

The study in electric brainwave began in 1875 by Richard Carton. The research focused on the characteristics of electric brainwave which affected to brain function in animals. It used a single electrode polarity on the cortex of the brain (cerebral cortex) and skull. Then, the potential of the electric brainwave occurred is measured using a 
galvanometer. In addition, the research found that the potential of the electric is increased while the animals sleep. It decreased and disappeared after the animals died. Another study was conducted by a physiologist named Adolf Beck in 1890 by measuring the electric potential of the cerebral cortex in dogs and rabbits. Several other scientists have tried to apply such tools used in the study of electric brainwaves. In 1914, Napoleon Cybulski and Jalenska Macieszyna recorded electric brainwaves of a dog. They then developed an amplifier to detect to detect brainwaves in human. It was applied first to humans in the 1920s by German neurologist Hans Berger [10]. EEG is a non-expensive, non-invasive and completely passive recording technique used in brainwave studies. Berger also discovered alpha waves where the results of this study has become the basis of detection and interpretation of electric brainwaves. Today, advanced brainwave recorder has been developed. This includes applications for detecting and analyzing electric brainwaves with higher accuracy and precision than in the past. However, advances in brainwave interpretation has been slow and needs a further research [11].

\section{Electroencephalogram (EEG)}

Brainwave signals can be divided into different types. Generally, brainwaves can be characterized by each signal's characteristics and method of recording. EEG is a measure of the electrical signal from the scalp or brain surface within the skull. The measured EEG signal is related to the brain or nerves in the sensing area. The EEG signal at certain frequencies reflects the mind and brainwaves as well as the measured signal at the facial muscles. The brain has a neuron, which can be contacted by transporting electrical particles through the cell in membrane. When a neuron is stimulated, it releases charged particles that travel along the nerve fibers which connected neurons. The next step is to stimulate the next nerve to release the charge. This electrical signal is known as a brainwave and can be recorded as electrical signals. The brainwaves' characteristic moves up and down to the general wave by using measurement of round per minute. There are several reasons why EEG is an exceptional tool for studying the neurocognitive processes underlying human behavior [12].

EEG has very high time resolution and captures cognitive processes in the time frame in which cognition occurs. EEG directly measures neural activity. EEG is inexpensive, lightweight, and portable. EEG monitors cognitive-affective processing in absence of behavioral responses show in Table I.

Table I. Types of brainwaves signals divided by frequency.

\begin{tabular}{|c|c|l|}
\hline $\begin{array}{c}\text { EEG } \\
\text { bands }\end{array}$ & $\begin{array}{c}\text { Frequency } \\
\text { (Hertz) }\end{array}$ & \multicolumn{1}{|c|}{ The relationship with the activity of the brain. } \\
\hline Gamma & $>30$ & The distinguished mind, fear, solving problems, learning \\
\hline Beta & $13-30$ & The body up or is triggered by various stimuli, the anxiety \\
\hline Alpha & $8-12$ & $\begin{array}{l}\text { Relax (but not sleepy), calm, concentrate in attentive } \\
\text { things }\end{array}$ \\
\hline Theta & $4-7$ & $\begin{array}{l}\text { In the imagine, concentrated mind, during REM sleep, } \\
\text { dream }\end{array}$ \\
\hline Delta & $1-3$ & In the deep sleep, no dream \\
\hline
\end{tabular}




\section{Panic Attacks}

Panic attacks can be severe or present uncomfortable experience to the sufferer within minutes. Often, patients of panic attacks are concerned about recurring attacks and the subsequent effects in public places. The fear of recurring attacks resulted in patients avoiding performing certain activities or going to public places, thus affecting one's daily life. For example, the patient may avoid exercising to keep the heart rate low in order to control the Panic Attacks symptoms. Panic attacks are generally intense for up to 10 minutes or less, and begin to decline. The severity of the condition can be heart disease, thyroid problems, respiratory distress and other illnesses. The Diagnostic and Statistical Manual of Mental Disorders, (DSM-IV-TR) [13] is a diagnostic criterion for mental disorders used in the United States Made by the United States Psychiatric Association. It provides diagnostic criteria which is characterized by at least 4 symptoms [14].

\section{Event-related potential (ERP)}

ERP is a small voltage that occurs in the brain structure in response to specific events or stimuli. The average of the electrical potential of the brain can be recorded from changes due to external or internal events that are triggered over the period of time [15]. ERP is studied to provide answers of the key questions in cognitive science [16]. The study found that there is the pattern of the sequence of stimulus. The brain electrical measurements are recorded as EEG. After that, each latency was brought to find the highest amplitude after stimulation. For example, the positive wave with the highest amplitude occurs at the time after stimulation about 100 milliseconds which is called P100, and if latency is about 200 or 300 milliseconds it was called P200, P300 [17].

P3 or P300 waves were discovered by [18] It is the brainwaves with highest amplitude occurring about 250 - 400 milliseconds after stimulation such as light, sound, or images of the Event-Related Potentials (ERP). This occurred at about 300650 milliseconds after the voltage level stimulation. It is compared with the next phases with P3 bandwidth in order to see the information of stimulation. There are several methods to retrieve P3 wave. One of the most common techniques used to detect P300 is oddball. This technique presents the sequences of repetitive stimuli which is infrequently interrupted by a different stimulus. The reaction of the participant to this oddball stimulus is recorded [19].

\section{Brainwave Detection Model for Panic Attacks}

This section presents the proposed model of brainwave detection for Panic Attacks based on event-related potential.

\section{Electroencephalogram (EEG) Detection}

The detection of brainwaves that comes through the signal capture device for brainwaves can capture the brainwave at the frequency from 1 to $80 \mathrm{~Hz}$. The frequency of EEG is shown in Table II. 
Table II. The frequency of the wave

\begin{tabular}{|c|c|}
\hline Raw Data & $\begin{array}{c}\text { Returns raw EEG, sampling rate at } \\
\mathbf{1 H z}\end{array}$ \\
\hline Delta & $1-3 \mathrm{~Hz}$ \\
\hline Theta & $4-7 \mathrm{~Hz}$ \\
\hline (Alpha1 )Low Alpha & $8-9 \mathrm{~Hz}$ \\
\hline (Alpha2 )High Alpha & $10-12 \mathrm{~Hz}$ \\
\hline (Beta1 )Low Beta & $13-17 \mathrm{~Hz}$ \\
\hline (Beta2 )High Beta & $18-30 \mathrm{~Hz}$ \\
\hline (Gamma1 )Low Gamma & $31-40 \mathrm{~Hz}$ \\
\hline (Gamma2 )High Gamma & $41-80 \mathrm{~Hz}$ \\
\hline Attention & integer value, between 0 and 100 \\
\hline Meditation & integer value, between 0 and 100 \\
\hline
\end{tabular}

However, the derived value of signal should be adjusted into the 1000 range form in order to obtained optimized range of signal. It is convenience to separate the signal value into specific type of signal. The adjusted formulas of each signal type are shown in Equation (1) below.

$$
\begin{gathered}
\theta=\frac{\theta}{1000} \\
\alpha=\frac{\left(\frac{\alpha_{\text {Low }}}{100}\right)+\left(\frac{\alpha_{\text {High }}}{100}\right)}{2} \\
\delta=\frac{\delta}{1000} \\
\beta=\frac{\left(\frac{\beta_{\text {Low }}}{100}\right)+\left(\frac{\beta_{\text {High }}}{100}\right)}{2} \\
v=\frac{\left(\frac{\gamma_{\text {Low }}}{100}\right)+\left(\frac{\gamma_{\text {High }}}{100}\right)}{}
\end{gathered}
$$

After the brainwaves has been collected, the waveform of HighBeta, LowBeta, HighGamma, LowGamma, with stimuli Event-related potential (ERP) has changed at

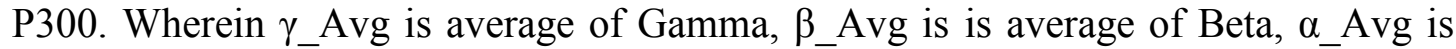
average of Alpha. The detected brainwave signals will change immediately and the visual attention was change with the highest frequency component of Gamma. According to the average value of Gamma signals, the Gamma have proportional constant, the study selects between 0.7 Gamma, beta and 0.3 Alpha were selected as calculating the value of the vision Panic, because the Gamma and beta is the highest mental tension value while Alpha mental tension state as a starting point. Shown in Equation (2).

Brainwaves Panic Attacks Measurement (BPAM)

$$
=\sqrt{\frac{\left(0.7 \times\left(\gamma_{A v g} \times \gamma^{2}+\beta_{A v g} \times \beta^{2}\right)+\left(0.3 \times \alpha_{A v g} \times \alpha^{2}\right)\right.}{\gamma_{A v g^{2}+} \beta_{A v g^{2}+}{ }^{\alpha} A v g^{2}}}
$$


When the signal detects through the brainwaves detection device, the pattern of Panic Attacks based on the brainwaves Panic Attacks equation will be used. The signal of alpha, beta and gamma will be evaluated. If the value of brainwaves Panic Attacks measurement is greater than 200, attention value is greater than 80 , and meditation is less than 20, then the Panic Attacks alerts. Figure 1 represents the algorithm of brainwaves Panic Attacks measurement.

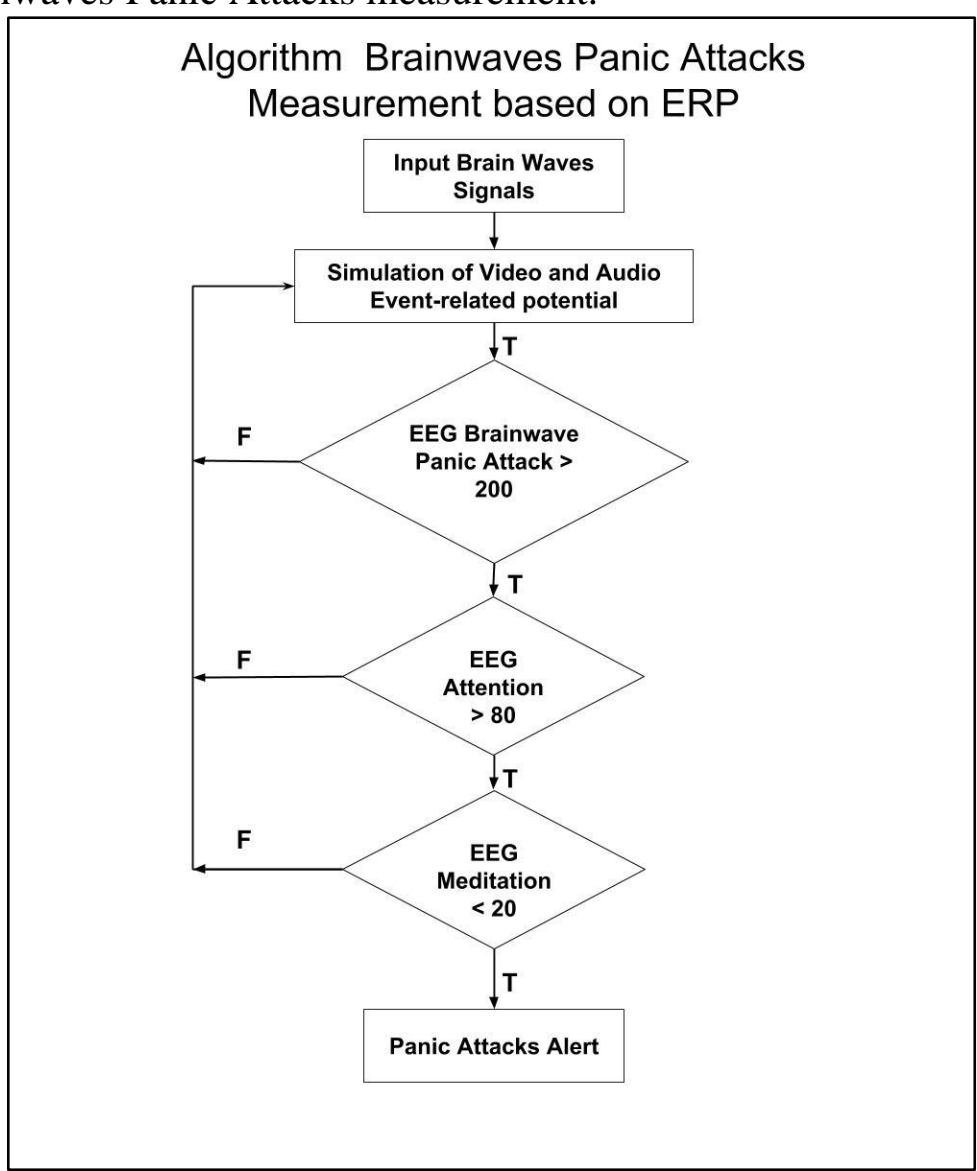

Figure 1. The algorithm of Brainwaves Panic Attacks Measurement Based on Event-related potential

\section{Research Methodology}

\section{The Experimental Design}

This research by proposed the model of brainwaves detection on Panic Attacks. The experiment was conducted the users in the simulation on thriller movie. The simulation implemented visual and audio stimuli in 30 users. The application of brainwaves detection has been developed and collected the data of users' brainwaves. During the experiment, the value of time, Delta, Theta, Alpha, HighBeta, LowBeta, HighGamma, LowGamma were recorded. Next, High and Low values are combined. Hence, the value to be used in the model will be Delta, Theta, Alpha, Beta, and Gamma.

The experimental group is 30 students aged from 19 to 21 with none or little experience with the device, and is balanced in terms of gender composition. The simulation used the video as media with storytelling in the initial stage in order to adjust the users' brainwaves. There will be frightening scene appearing at almost the end of the video. 
As shown is Figure 2, a zombie appears at the end of the video with sound effects. This will terrify users and cause the changes in EEG during Event-related potential environment. The framework is shown in Figure 3.

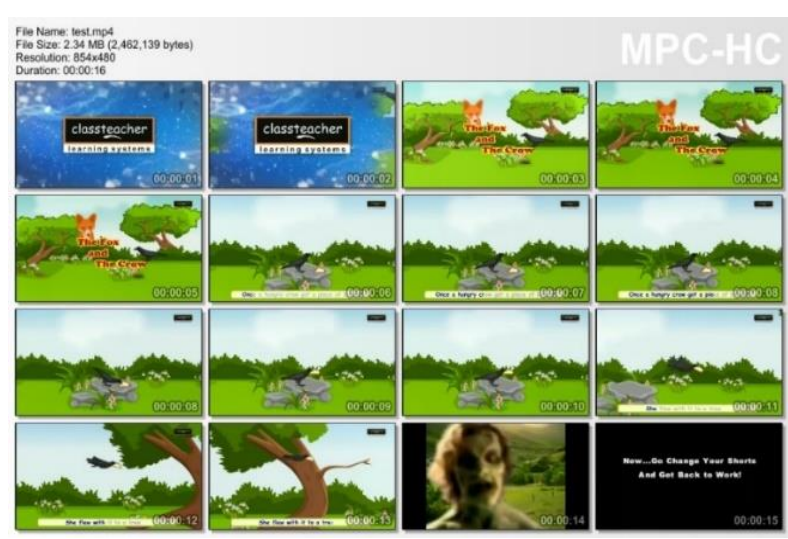

Figure 2. Video used to test for brainwaves patterns during Panic Attacks

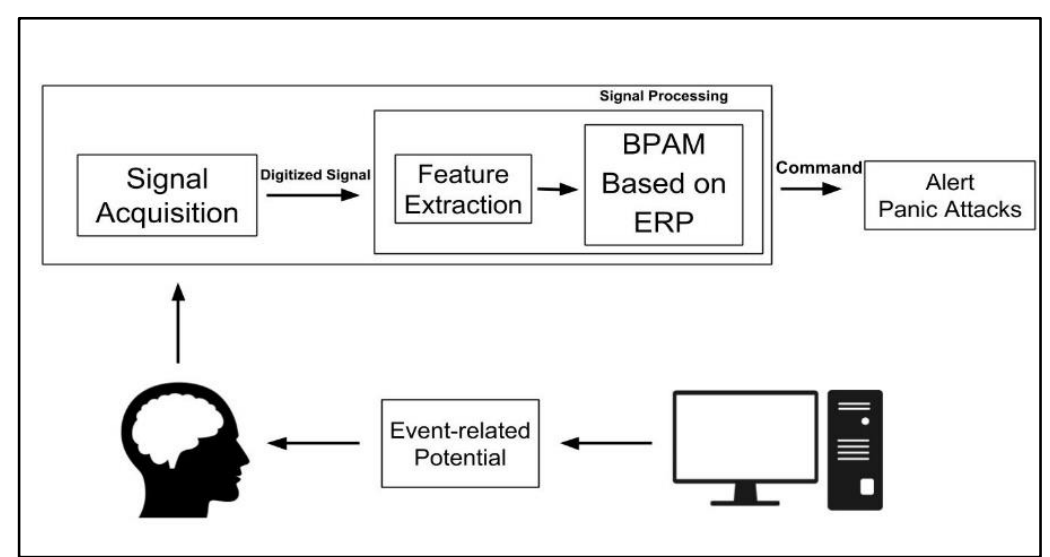

Figure 3. Framework Model Panic Attacks Based on Event-related Potential

\section{Panic Attacks Measurement Analysis}

After the experiment, the recorded EEG data of each user will be compared with user's questionnaire to consider the consistency of the measurement. The BAI questionnaire used in this experiment consisted of 21 multiple-choice questions. This can be used to measure the severity of anxiety in children and adults. Users need to answer about common symptoms of anxiety such as sweating without heat or fear of something happened. The answer will be calculated to determine the score which is based on a scale of value 0-9 is minimal anxiety. 10-18 is mild anxiety. 19-29 is moderate anxiety. 30-63 is severe anxiety. Score 19-63 is mild anxiety and moderate anxiety has been suggested to detect clinically significant symptoms [20]. If the comparison between EEG data and questionnaire results are the same, it can be referred as the Panic Attacks symptoms.

\section{Experimental Results}

The results of human brainwaves detection while frightened by associated stimulation based on Event-related potential are shown in Figure 4. There are 30 participants in the experiment. The average value of participants' Brainwaves Panic Attacks measurement (BPAM) is at around 220 when a stimuli is presented. However, the results found that there is one case that the brainwaves are different 
from others. This leads to Panic Attacks symptoms for these participants. The steep graph during 23-26 time unit in the experiment represents those of Case 1. It can be seen in Figure 5. In addition, the Brainwaves Panic Attacks Measurement (BPAM) for these cases are the highest at around 420.

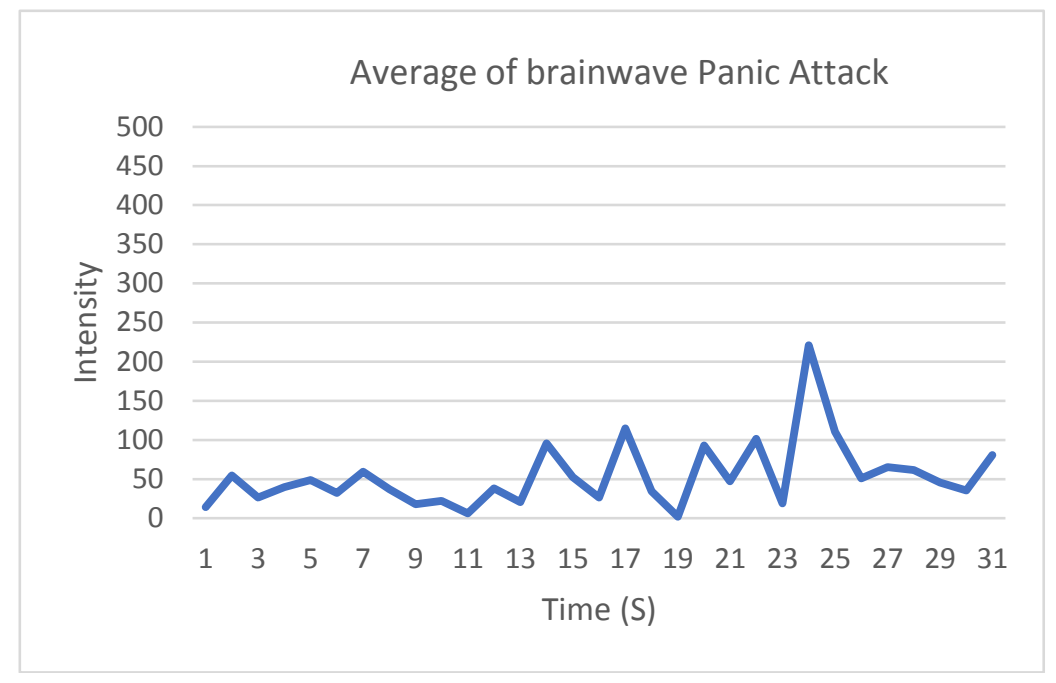

Figure 4. The average of brainwaves Panic Attacks of 30 participants after being Stimulated by Event - related potential (ERP).

It can be seen that the fluctuation of BPAM are sideway trend. Nevertheless, Event-Related Potential (ERP) are added and there is a change of BPAM significantly with stimulation on pictures and sound. In addition, the BPAM values with ERP have risen up immediately. Compared to the Beck Anxiety Inventory (BAI) score, if the BPAM score is up to 420 , it is considered as moderate anxiety.

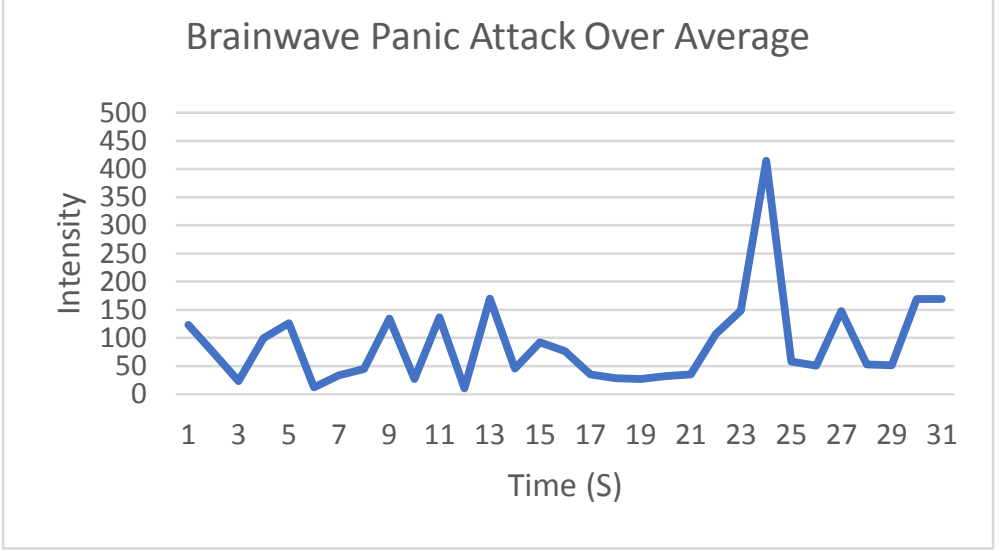

Figure 5. Brainwave Panic Attack of the person who made the BAI questionnaire and Scored 10-29 after passing the simulation Event-related potential

The graph in Figure 6 shows the comparison between normal cases and different cases of average BPAM. It appeared that there is significant difference in BPAM which is derived from recorded EEG. To consider the risk of Panic Attacks in participants, the attention value is also included in the experiment for this case. Figure 7 shows the attention value for suspected cases found in the experiment. The value of the attention of these cases are significantly higher than the normal cases. This leads to unable to control their mind as usual and which can be seen from the body effects. 
Table 3 shows BPAM and BAI score from the participants. The BPAM score for normal cases are from 80 to 400 and BAI score of this group is between 0 and 18 . The suspected to be Panic Attacks 1 cases have a BAI score more over 19 moderate to severe anxiety which BAI score 19-63 and BPAM from 401 to 1000 and BAI score at the third level group has been suggested to detect clinically significant symptoms.

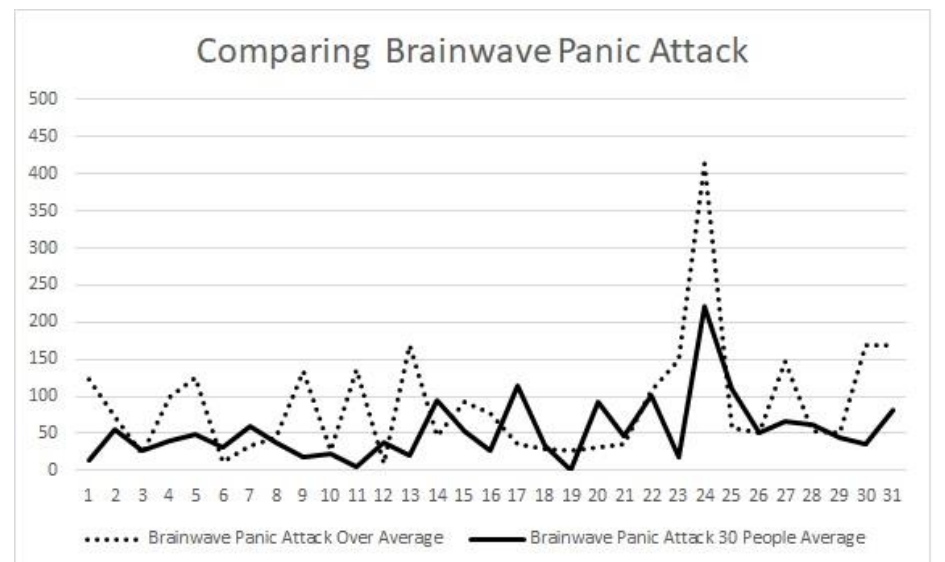

Figure 6..Comparison of Brainwave Panic Attacks Between Normal Cases and Different Cases

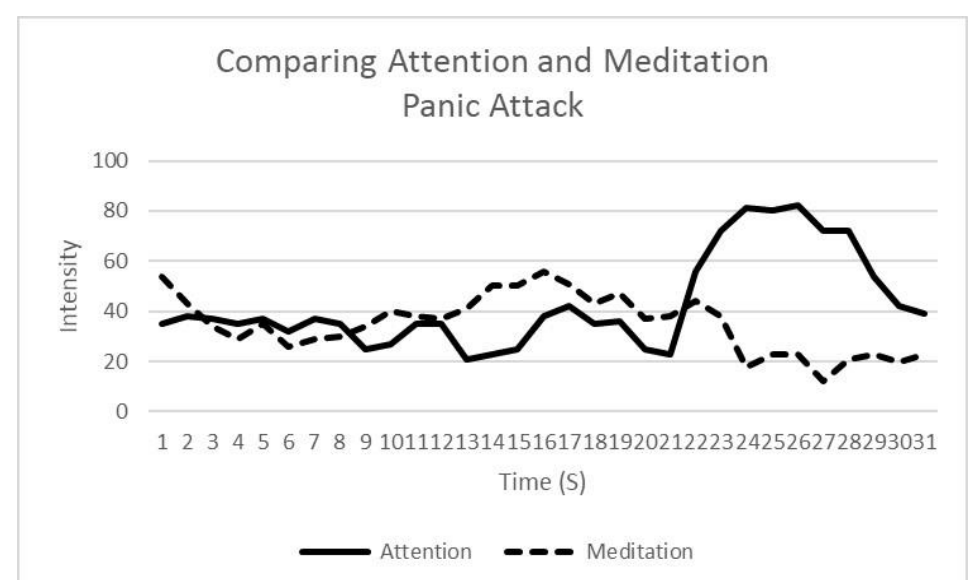

Figure 7. The visual state for brainwave signals from Panic Attack

Table III.

The Comparison of BAI Measurement With Brainwave Panic Attacks

\begin{tabular}{|c|c|c|}
\hline BAI Score & Total Student & BPAM Score \\
\hline 0-9 normal to minimal anxiety & 22 & $80-200$ \\
\hline $\begin{array}{c}\text { 10-18 mild to moderate } \\
\text { anxiety }\end{array}$ & 7 & $201-400$ \\
\hline $\begin{array}{c}\text { 19-29 moderate to severe } \\
\text { anxiety }\end{array}$ & 1 & $401-600$ \\
\hline $30-63$ severe anxiety & - & $601-1000$ \\
\hline
\end{tabular}




\section{Conclusion}

The symptoms of panic attack impact daily lives and it can also increase the risk to other consequences. Basically, panic attacks can come from external observation only. A questionnaire by psychiatric tests is used to observe the symptom. This research proposed the model of brainwaves detection for panic attacks and evaluation of the level of the symptoms based on Event-related potential (ERP). The model can measure the level of symptoms Panic Attack using the changes in brainwaves occurred during the simulation with pictures and sound on the experimental group of 30 people. The survey related to symptoms has been used regarding to the criteria of The Beck Anxiety Inventory (BAI).

The research is measured by the EEG brainwaves from studying brainwaves during Panic Attacks, when human's panic and fear arise. Those are considered as the Panic Attacks. In the experiment, the panic may be more shocked than usual, and it is measured as a level of The Beck Anxiety Inventory (BAI) based on the criteria. The level is from 1 to 4 . The level 1 is minimal anxiety. The second level is mild anxiety. The third level is considered as moderate anxiety and level 4 is severe anxiety. This research can also detect and measure the brainwaves with Event-related potential directly in order to create the Panic Attacks brainwave model. There are 22 out of 30 people in the experiment showed the brainwaves based on Gamma and Beta wavelength using BPAM score between 80 and 200. It is considered as minimal anxiety, whereas 7 people showed the BPAM score during 201 to 400 . There is only one person that the BPAM score was more than 400. This is moderate anxiety. The level of severe anxiety is not found in the experiment group. Therefore, those 1 people can be considered having Panic Attacks symptoms has been suggested to detect clinically significant symptoms

The research also presents that there are changes of the Beta and Gamma wave length significantly. For the further analysis, it found that the brainwave pattern for the

meditation had decreased but the attention had increased. The change occurred in wave P300 which associated to external stimuli by relationship with Event-related potential (ERP).

For future works, the source and various types of content could have included in the experiment to detect on the Panic Attack brainwaves. This is to find the different situation with regards to different of brainwaves pattern on the Panic Attack brainwaves detection. The future experiment may implement virtual reality technology for more realistic situations. The medical records and pre-test of Panic Attacks can be included in the primary stage of the next experiment to filter the specific types of panic and fear. This can help more to increase accuracy in the level of brainwaves related to Panic Attacks.

\section{Conflict of Interests.}

There are non-conflicts of interest.

\section{References}

[1] S. K. Raman, J. K. Jayaram, S. Murugan, A. Saha, and R. K. Kavitha, "Design of a robust method to acquire EOG signals using Bio-medical signal processing," in 2016 Online International Conference on Green Engineering and Technologies (IC-GET), 2016, pp. 1-4. 
[2] A. Yildirim, "Analysis and classification of spelling paradigm EEG data and an attempt for optimization of channels used," Ph.D. dissertation, Middle East Technical University, 2010.

[3] L. Grossman. "The Invention Issue Best Inventions", TIME MAGAZINE, vol.178, no.21, 2011. [Online]. Available:

http://content.time.com/time/covers/0,16641,20111128,00.html.

[4] H. Zhang, and J. Horsman. "Puzzlebox Bloom Premiere at CES 2015," 2015 International CES. 2015. [Online]. Available: https://puzzlebox.io/post/puzzlebox-bloom-premiere-ces-2015/.

[5] P. S. Kanagasabai, R. Gautam, and G. N. Rathna, "Brain-computer interface learning system for Quadriplegics," in 2016 IEEE 4th International Conference on MOOCs, Innovation and Technology in Education (MITE), 2016, pp. 258-262.

[6] A. Kubacki, A. Jakubowski, and D. Rybarczyk, "Research on possibilities of transporter movement using brain-computer interface based on Steady-State Visually Evoked Potential (SSVEP)," in 2017 22nd International Conference on Methods and Models in Automation and Robotics (MMAR), 2017, pp. 218-221.

[8] Y. Hao, Z. Xu, J. Wang, Y. Liu, and J. Fan, "An approach to detect crowd panic behavior using flow-based feature," in 2016 22nd International Conference on Automation and Computing (ICAC), 2016, pp. 462-466.

[9] M. Laytrakul \& P. Suksanit, "Panic disorder", Ramathibodi Hospital, Mahidol University, 2011. Retrieved January 21, 2018, from https://med.mahidol.ac.th/ramamental/generaldoctor/panic-disorder-2/.

[10] Jung, \& Berger, "Fiftieth anniversary of Hans Berger's publication of the electroencephalogram His first records in 1924-1931," Journal of Psychiatry and Nervous Diseases, vol.227, 279-300, 1979.

[11] S. Finger, Origins of neuroscience: a history of explorations into brain function. Oxford University Press, USA, 2001.

[12] M. X. Cohen, "It's about time," Front. Hum. Neurosci., vol. 5, p. 2, 2011. Available:

https://www.ncbi.nlm.nih.gov/pmc/articles/PMC3025647/ [Accessed: January 21, 2018].

[13] G. R. Norton, B. Harrison, J. Hauch, and L. Rhodes, "Characteristics of people with infrequent panic attacks.," J. Abnorm. Psychol., vol. 94, no. 2, p. 216, 1985.

[14] M. Smith, M.A., L. Robinson, and J. Segal, "Panic Attacks and Panic Disorder," 2018. Available: https://www.helpguide.org/articles/anxiety/panic-attacks-andpanic-disorders.html [Accessed: January 21, 2018].

[15] D. H. R. Blackwood and W. J. Muir, "Cognitive brain potentials and their application,” Br. J. Psychiatry, vol. 157, no. S9, pp. 96-101, 1990. 
[16] E. K. Vogel and S. J. Luck, "The visual N1 component as an index of a discrimination process," Psychophysiology, vol. 37, no. 2, pp. 190-203, 2000.

[17] S. J. Luck. An Introduction to the Event-Related Potential Technique. Cambridge: The MIT Press. ISBN 0-262-12277-4. 2005.

[18] S. Sutton, M. Braren, J. Zubin, and E. R. John, "Evoked-potential correlates of stimulus uncertainty," Science (80-. )., vol. 150, no. 3700, pp. 1187-1188, 1965.

[19] R. van Dinteren, M. Arns, M. L. A. Jongsma, and R. P. C. Kessels, "P300 development across the lifespan: a systematic review and meta-analysis," PLoS One, vol. 9, no. 2, p. e87347, 2014.

[20] L. J. Julian, "Measures of anxiety: state-trait anxiety inventory (STAI), Beck anxiety inventory (BAI), and Hospital anxiety and Depression scale-anxiety (HADS-A)," Arthritis Care Res. (Hoboken), vol. 63, no. S11, pp. S467-S472, 2011. 